\title{
Parathyroid carcinoma coexisting with multiple parathyroid adenomas: a case report
}

\author{
Masao Takenobu, Sueyoshi Moritani, Katsuyuki Kawamoto, Kana Yoshioka and Hiroya Kitano
}

Kusatsu General Hosipital, Otolaryngology Head and Neck Thyroid Surgery, Shiga 525-8585, Japan

\begin{abstract}
Primary hyperparathyroidism is usually caused by parathyroid adenoma; however, parathyroid carcinoma is a rare cause. We report a rare case of 74-year-old female of primary hyperparathyroidism caused by parathyroid carcinoma (PC) and coexisting multiple parathyroid adenomas. She was referred to our hospital for primary hyperparathyroidism and a suspected thyroid tumor. She had no family history of malignant tumor. Computed tomography (CT) and ultrasonography of the neck revealed some masses posterior to both thyroid lobes. Those masses were believed to be parathyroid lesions. However, another mass located posterior to the right upper thyroid lobe seemed to be heterogeneous, which indicated a malignant thyroid tumor as well as parathyroid tumor. The preoperative diagnosis was multiple parathyroid adenoma and suspicious incidental thyroid carcinoma. Therefore, the patient underwent total parathyroidectomy and thyroidectomy. The histopathological diagnosis was parathyroid carcinoma coexisting with multiple parathyroid adenomas. There was no evidence of recurrence at 1 year after the surgery. It was difficult to diagnose PC preoperatively. Few rare cases of PC coexisting with parathyroid adenoma in multiple endocrine neoplasia type 1 (MEN1) have been reported. Therefore, careful follow-up was necessary considering the possibility of MEN1, though she did not wish for a genetic examination.
\end{abstract}

Key words: Parathyroid carcinoma, Parathyroid adenoma, Multiple parathyroid adenoma, Primary hyperparathyroidism

PARATHYROID CARCINOMA (PC) is a rare malignancy observed in $<1 \%$ of primary hyperparathyroidism cases [1]. PC usually occurs as a sporadic disease but is also associated with genetic syndromes such as hyperthyroidism, jaw syndrome, or multiple endocrine neoplasm type 1 (MEN1) [2-5]. Conversely, parathyroid adenoma is the most common cause of hyperparathyroidism (87\%-91\%), and the incidence of multiple parathyroid adenomas is $2 \%-15 \%$ of all parathyroid adenomas $[6,7]$. The coexistence of PC and adenoma is extremely rare. We herein report a case of PC coexisting with multiple parathyroid adenomas.

\section{Case report}

A 74-year-old female was referred to our hospital for primary hyperparathyroidism and a suspected thyroid tumor. She complained of osteoporosis and urinary stone. She had no particular family history of malignant tumor. Laboratory test results were as follows: calcium, $14.8 \mathrm{mg} / \mathrm{dL}$; albumin, $3.9 \mathrm{~g} / \mathrm{dL}$; phosphorus, $1.9 \mathrm{mg} / \mathrm{dL}$;

Submitted Mar. 16, 2020; Accepted May 1, 2020 as EJ20-0139 Released online in J-STAGE as advance publication May 30, 2020 Correspondence to: Masao Takenobu, Kusatsu General Hospital, Otolaryngology Head and Neck Thyroid Surgery, 1660 Yabase, Kusatsu, Shiga, 525-8585, Japan.

E-mail: masaotakenobu@yahoo.co.jp intact parathyroid hormone, $1,039 \mathrm{pg} / \mathrm{mL}$; free $\mathrm{T} 3,2.70$ $\mathrm{pg} / \mathrm{mL}$; free T4, $0.74 \mathrm{ng} / \mathrm{dL}$; thyroglobulin, $12.53 \mathrm{ng} / \mathrm{mL}$; CEA, $5.6 \mathrm{ng} / \mathrm{mL}$; and calcitonin, $1.24 \mathrm{pg} / \mathrm{mL}$.

Computed tomography (CT) examination revealed a mass in the posterior thyroid lobe and on the right side of the thyroid gland (Fig. 1a).

Technetium-99 m methoxy-isobutyl-isonitrile (Tc-99 $\mathrm{m}$ MIBI) parathyroid scintigraphic examination revealed an increase in the late phase focal activity adjacent to both lower poles (Fig. 1b).

Ultrasonography of the neck revealed an $11-\times 13-$ $\mathrm{mm}$-sized mass located inferior to the lower part of the right thyroid lobe (Fig. 2a), 11- $\times 11$-mm-sized mass located inferior to the lower part of the left thyroid lobe (Fig. 2b), and 7.0- $\times 12$-mm-sized mass located behind the upper left thyroid lobe (Fig. 2c). The masses were hypoechoic with smooth and clear borders, indicating parathyroid lesions. Moreover, ultrasonography revealed another hypoechoic mass located posterior to the right thyroid lobe (Fig. 2d). The size of this lesion was $19 \times$ $14 \mathrm{~mm}$ (depth-width ratio; 1.36). The boundary between the mass and thyroid was unclear and color Doppler mode demonstrated normal vascularity. Based on these findings, the mass was suspected to be a malignant thyroid or parathyroid tumor. Therefore, fine-needle aspiration cytology (FNAC) was performed to characterize this 


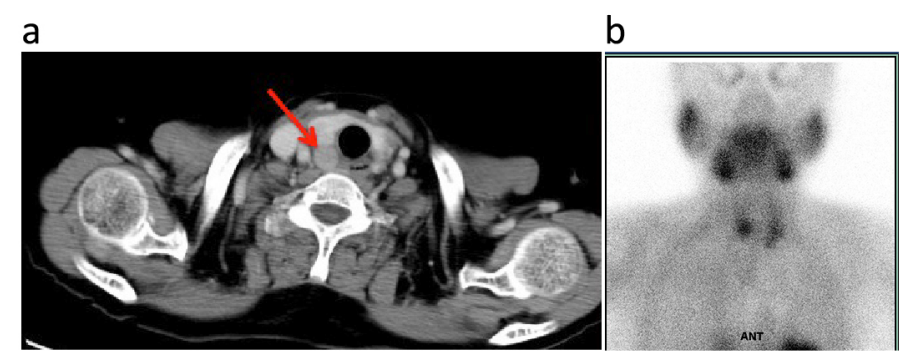

Fig. 1 a: CT image of the right posterior thyroidal mass (indicated by an arrow).

b: Technetium-99 m methoxy-isobutyl-isonitrile scintigram showed an increase in late phase focal activity adjacent to both lower poles.

a

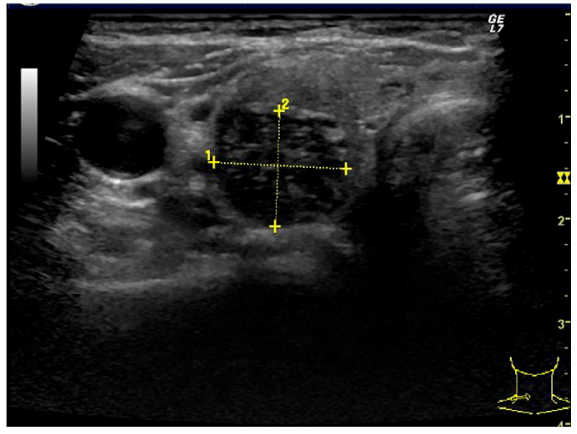

C

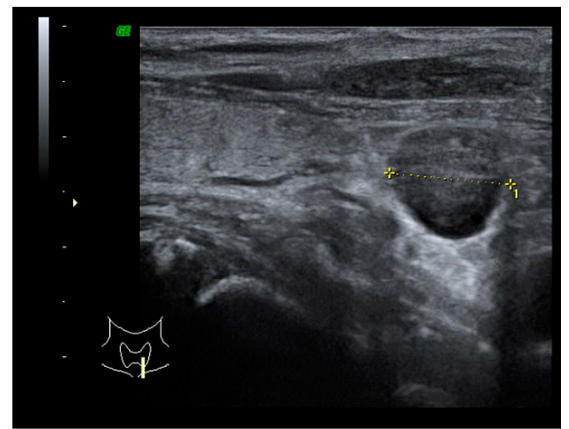

b

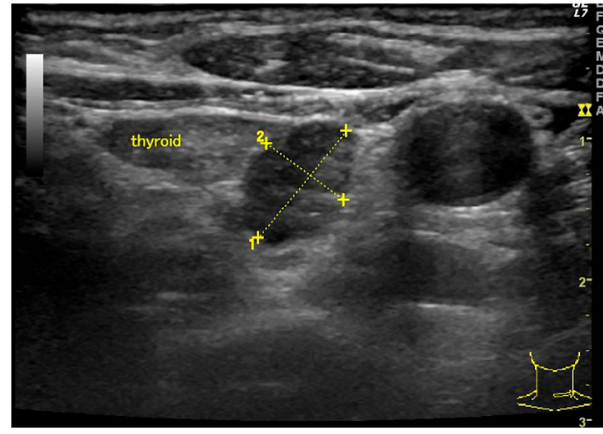

d

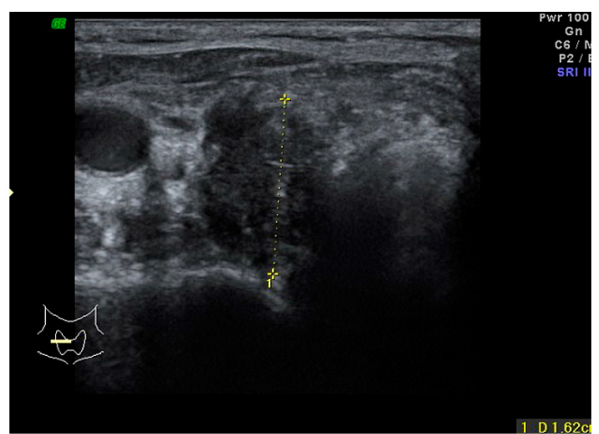

Fig. 2 a: Neck ultrasonography showing the right lower parathyroid tumor.

b: Left lower parathyroid tumor.

c: Left upper parathyroid tumor.

d: The mass located posterior to the right thyroid lobe.

suspicious lesion.

FNAC results revealed a medullary carcinoma with intranuclear cytoplasmic inclusions and an amyloid-like structure. The serum calcitonin level was $1.24 \mathrm{pg} / \mathrm{mL}$ (normal range, $0.0-3.91 \mathrm{pg} / \mathrm{mL}$ ) and CEA was 5.6 $\mathrm{ng} / \mathrm{mL}$ (normal range, $0.0-6.0 \mathrm{ng} / \mathrm{mL}$ ). Based on these symptoms, primary hyperparathyroidism with multiple parathyroid adenomas and suspicious incidental thyroid carcinoma was diagnosed.

The patient underwent total parathyroidectomy and total thyroidectomy (Fig. 3a, b). During the surgery, the lesion that was suspected to be a thyroid tumor adhered to the surrounding tissue and it seemed to be an extrathyroidal lesion. However, it was difficult to determine whether the lesion was a lymph node or a superior right parathyroid tumor. Because of the adhesion, preservation of the right recurrent laryngeal nerve required substantial efforts. Other lesions which were suspected to be parathyroid tumors were easy to remove without any adhesions.

\section{Histopathological diagnosis}

There were no findings that indicated medullary carcinoma or other malignancies in the resected thyroid. The specimens of the right inferior, left superior, and left inferior parathyroid contained normal thyroid rim. They comprised poorly atypical glandular epithelial cells with a pale eosinophilic endoplasmic reticulum growing in 


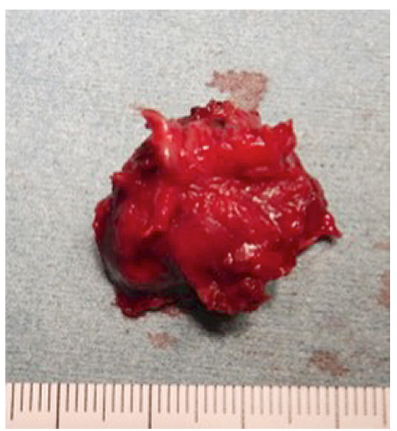

b

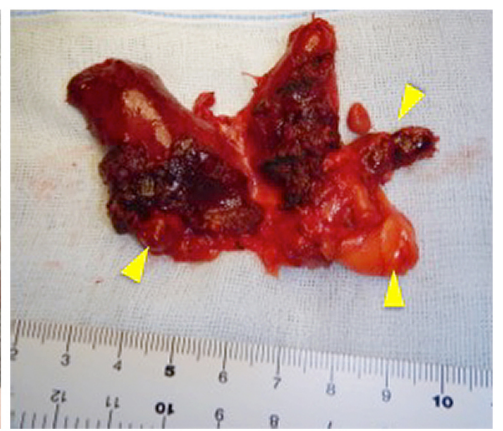

Fig. 3 a: Extrathyroidal heterogeneous lesion located posterior to the right lobe.

b: The resected thyroid and suspicious parathyroid adenomas (arrows).

a

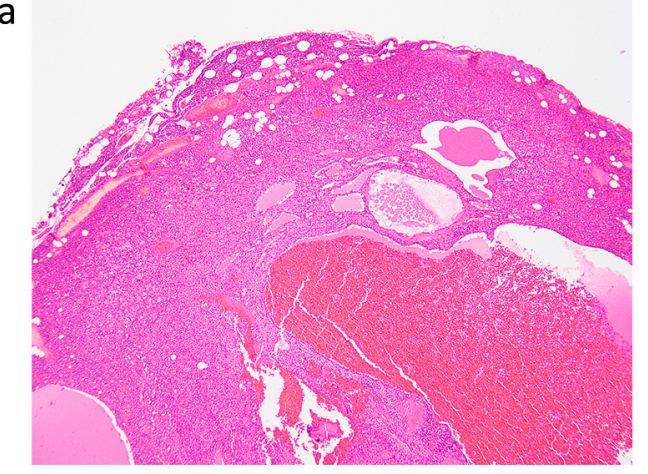

C

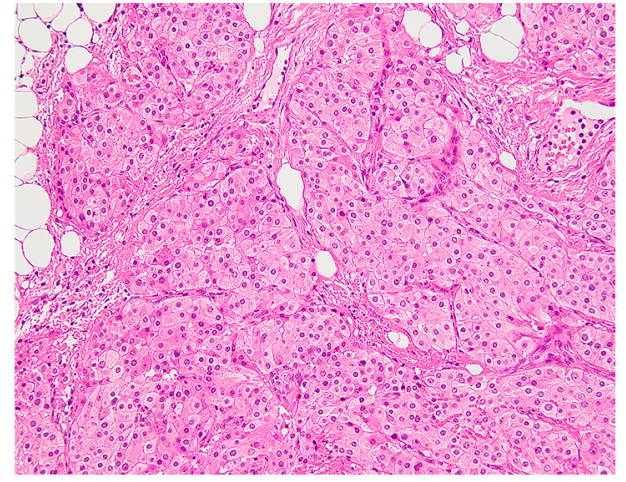

b

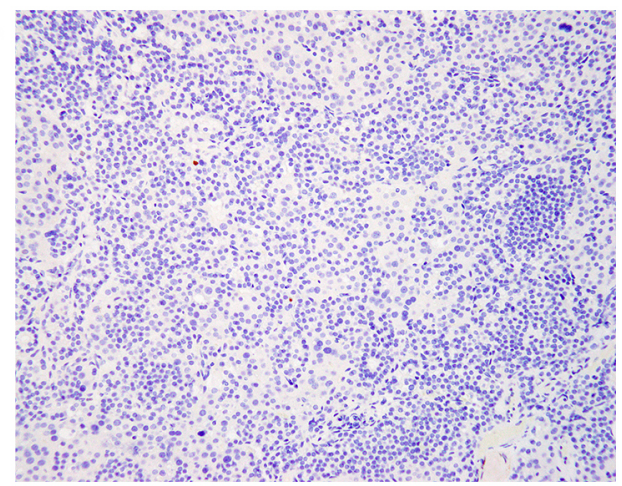

d

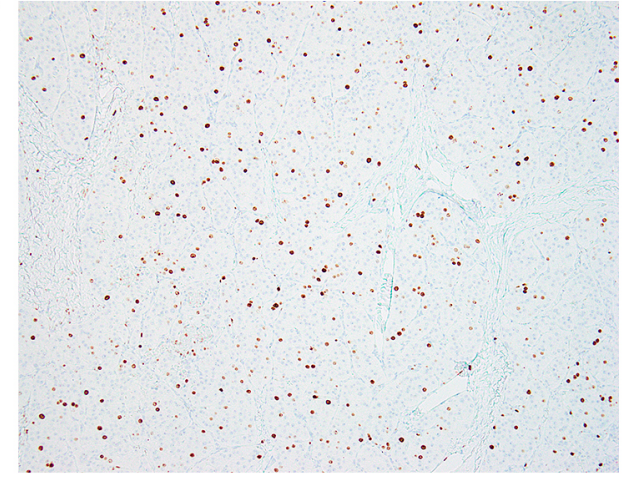

Fig. 4 a: Histopathological findings of the parathyroid adenoma [Hematoxylin-Eosin staining (HE), magnification: 40×]

b: Immunohistochemical analysis by Ki67 of parathyroid adenoma $(100 \times)$.

c: Histopathological findings of the parathyroid carcinoma (HE staining, magnification: $200 \times$ )

d: Immunohistochemical analysis by Ki67 of parathyroid carcinoma (magnification: 100×).

solid, funicular, tubular, and cystic forms, which were diagnosed as parathyroid adenomas with the following characteristics: parathyroid hormone (PTH)-positive, GATA3-positive, and Ki67-labeling index (LI) of $<1 \%$ as demonstrated by immunohistochemical analysis (Fig. 4a, b). The extrathyroidal lesion that was initially difficult to identify was not a thyroid tumor or lymph node but a right superior parathyroid with glandular epithelial cells containing round nuclei showing solid and alveolar growth. However, this specimen exhibited extracapsular invasion and necrosis, which was different from the characteristics of the other resected parathyroid adenomas. Immunohistochemical analyses revealed that this specimen was PTH-positive, GATA3-positive, and Ki67LI of $>10 \%$. Based on these results, this lesion was determined to be a PC (Fig. 4c, d). The pathology committee consulted with the Japanese Association of Endocrine Surgery and approved the diagnoses.

At 2 months after the surgery, the serum calcium and phosphate levels were normal, and positron emission 
tomography-CT showed no signs of 18-FDG accumulation indicating distant metastases. Furthermore, there was no evidence of recurrence at 1 year after the surgery.

\section{Discussion}

$\mathrm{PC}$ is a rare malignancy found in $<1 \%$ cases of primary hyperparathyroidism [1]. PCs are usually hormonally active, whereas $10 \%$ of them are nonfunctional [8-10]. Preoperative diagnosis of PC is often difficult because there are no specific clinical features to distinguish PC cases from parathyroid adenomas, except in cases with distant metastases or symptoms such as hoarseness caused by the recurrent laryngeal nerve invasion.

In a previous report, the average serum calcium levels in patients with PC were higher $(15.9 \mathrm{mg} / \mathrm{dL})$ than the calcium levels reported in patients with parathyroid adenomas $(12 \mathrm{mg} / \mathrm{dL})$. Only $10 \%$ of patients with PC had serum calcium levels of $<13 \mathrm{mg} / \mathrm{dL}$, and $75 \%$ of patients had serum calcium of $\geq 14 \mathrm{mg} / \mathrm{dL}$ [11]. In our case, the preoperative serum calcium level was $14.8 \mathrm{mg} / \mathrm{dL}$, which may indicate malignancy.

The mean depth-width (DW) ratio of the parathyroid carcinoma lesion was reported to be 1.21 using ultrasonography, which was significantly higher than that of parathyroid adenoma [12]. In this study, the DW ratio of the right upper lesion was 1.36; however, it may not be a specific finding to distinguish PC from adenoma compared with distinguishing $\mathrm{PC}$ from other parathyroid lesions.

Generally, preoperative FNAC of the parathyroid is not recommended owing to the high possibility of a false-negative result and the threat of dissemination [8, 13]. However, in our case, the lesion located posterior to the right thyroid lobe had obviously different findings from the other lesions that indicated parathyroid adenoma and was difficult to distinguish from the other thyroid tumors. Therefore, we performed the FNAC on this heterogeneous lesion, which led to a suspicion of medullary thyroid carcinoma (MTC). If the tumor was identified to be MTC, the possibility of the tumor being multiple endocrine neoplasia type 2 (MEN2) had to be considered. However, the diagnosis of MTC using FNAC is often difficult because of its low incidence, various cellular morphologies, non-typical cell shapes, and low cellularity. Hence, the diagnostic accuracy of MTC by FNAC is low compared with measuring calcitonin in the wash-out fluid from the aspiration needle or preoperative immunocytochemical examination using an anticalcitonin antibody $[14,15]$. In this case, the normal serum calcitonin and CEA levels did not support the possibility of MTC. Therefore, we suspected a malignant thyroid tumor coexisting with multiple parathyroid adenomas rather than MEN2. However, retrospectively, measuring the highly sensitive parathyroid hormone in the wash-out fluid from the aspiration needle might be helpful to distinguish parathyroid and thyroid tumors.

Surgical treatment is the only curative method for PC. Generally, en bloc resection of PC along with the ipsilateral thyroid lobe and adjacent structures is recommended for improving local disease control and significantly increasing long-term survival [16].

In our case, total parathyroidectomy and total thyroidectomy were performed because of the suspicion of coexisting malignant thyroid tumors before the surgery. The right superior lesion was a possible metastatic lymph node caused by thyroid carcinoma during surgery. This is why we performed total thyroidectomy and not hemithyroidectomy.

Consequently, there might not be sufficient surgical margins, particularly the surrounding tissue of the recurrent laryngeal nerve. Although the efficacy of adjuvant radiation therapy for treating $\mathrm{PC}$ has not been proven, Munson et al. reported the potential benefit of adjuvant radiation therapy in treating PC [17]. Therefore, adjuvant radiation therapy may be one of the favorable options. However, in our case, the patient did not require any additional treatments. Although there was no recurrence of the tumor at 1 year after the surgery, careful observation is needed.

The postoperative pathological diagnoses were PC coexisting with multiple parathyroid adenomas.

Double adenomas are observed in $<5 \%$ of primary hyperparathyroidism cases [18]. The coexistence of PC with multiple adenomas is believed to be extremely rare, although cases of double PC were reported in some previously published studies, one of which focused on patient with MEN1 [3, 19].

Hyperparathyroidism associated with MEN1 is usually caused by parathyroid hyperplasia, whereas $\mathrm{PC}$ is a rare causative lesion [5]. In previous reports, there were few rare cases of $\mathrm{PC}$ coexisting with hyperplasia or adenoma in patients with MEN1 [3, 5, 20]. Therefore, MEN1 could be considered in our case. The patient had no family history and no other tumors such as pituitary or pancreatic tumors that could be components of MEN1. Furthermore, $90 \%$ of the parathyroid lesions in patients with MEN1 occur in patients aged approximately 40 years and our patient was 74 year old [21, 22]. Despite these observations, the possibility of MEN1 without family history could not be completely excluded because the patient did not undergo a genetic examination to confirm the diagnosis. In fact, $8 \%-14 \%$ of patients with MEN1 are reported to have the non-familial form [23].

Therefore, we considered the possibility of MEN1 and 
decided to follow up with the patient to prevent the recurrence of the $\mathrm{PC}$ and the asynchronous tumor.

In conclusion, it was difficult to preoperatively diagnose PC in our case. The intraoperative findings of PC were different enough from those of the adenoma to suspect a malignancy. The limitation of our report is that we could not perform the genetic screening for MEN1. Our case of PC coexisting with multiple parathyroid adeno- mas was indeed rare, even if these tumors were associated with MEN1.

\section{Disclosure Statement}

None of the authors have any potential conflicts of interest associated with this report.

\section{References}

1. Marcocci C, Cetani F (2011) Clinical practice. Primary hyperparathyroidism. N Engl J Med 365: 2389-2397.

2. Piciu D, Piciu S, Barbus E, Pestean C, Larg MI, et al. (2016) Primary hyperparathyroidism-Jaw tumor syndrome: a confusing and forgotten diagnosis. Med Pharm Rep 89: 555-558.

3. Omi Y, Horiuchi K, Haniu K, Tokura M, Nagai E, et al. (2018) Parathyroid carcinoma occurred in two glands in multiple endocrine neoplasia 1: a report on a rare case. Endocr J 65: 245-252.

4. del Pozo C, Garcia-Pascual L, Balsells M, Barahona MJ, Veloso E, et al. (2011) Anglada-barcel. Parathyroid carcinoma in multiple endocrine neoplasia type 1 . Case report and review of the literature. Hormones (Athens) 10: 326331.

5. Christakis I, Busaidy NL, Cote GJ, Williams MD, Hyde SM, et al. (2016) Parathyroid carcinoma and atypical parathyroid neoplasms in MEN1 patients; a clinicopathologic challenge. The MD Anderson case series and review of the literature. Int J Surg 31: 10-16.

6. Piggott RP, Waters PS, Ashraf J, Colesky F, Kerin MJ (2013) Water-clear cell adenoma: a rare form of hyperparathyroidism. Int J Surg Case Rep 4: 911-913.

7. Kuhel WI, Gonzales D, Hoda SA, Pan L, Chiu A, et al. (2001) Synchronous water-clear cell double parathyroid adenomas: a hitherto uncharacterized entity? Arch Pathol Lab Med 125: 256-259.

8. Cetani F, Frustaci G, Torregrossa L, Magno S, Basolo F, et al. (2015) A nonfunctioning parathyroid carcinoma misdiagnosed as a follicular thyroid nodule. World J Surg Oncol 13: 270.

9. Wang L, Han D, Chen W, Zhang S, Wang Z, et al. (2015) Non-functional parathyroid carcinoma: a case report and review of the literature. Cancer Biol Ther 16: 1569-1576.

10. Dikmen K, BostanciH, Gobut H, Yildiz A, Ertunc O, et al. (2017) Nonfunctional double parathyroid carcinoma with incidental thyroid micropapillary carcinoma: a rare case. Pan Afr Med J 27: 241.

11. Fernandes JMP, Paiva C, Correia R, Polónia J, Moreira da Costa A (2018) Parathyroid carcinoma: from a case report to a review of the literature. Int J Surg Case Rep 42: 214217.

12. Hara H, Igarashi A, Yano Y, Yashiro $\mathrm{T}$, Ueno E, et al.
(2001) Ultrasonographic features of parathyroid Carcinoma. Endocr J 48: 213-217.

13. Kim J, Horowitz G, Hong M, Orsini M, Asa SL, et al. (2017) The dangers of parathyroid biopsy. J Otolaryngol Head Neck Surg 46: 4.

14. Suzuki A, Hirokawa M, Takada N, Higuchi M, Ito A, et al. (2017) Fine-needle aspiration cytology for medullary thyroid carcinoma: a single institutional experience in Japan. Endocr J 64: 1099-1104.

15. de Crea C, Raffaelli M, Maccora D, Carrozza C, Canu G, et al. (2014) Calcitonin measurement in fine-needle aspirate washouts $v s$. cytologic examination for diagnosis of primary or metastatic medullary thyroid carcinoma. Acta Otorhinolaryngol Ital 34: 399-405.

16. Holmes EC, Morton DL, Ketcham AS (1969) Parathyroid carcinoma: a collective review. Ann Surg 169: 631-640.

17. Munson ND, Foote RL, Northcutt RC, Tiegs RD, Fitzpatrick LA, et al. (2003) Parathyroid Carcinoma: is there a role for adjuvant radiation therapy? Cancer 98: $2378-2384$.

18. Harness JK, Ramsburg SR, Nishiyama RH, Thompson NW (1979) Multiple adenomas of the parathyroids: do they exist? Arch Surg 114: 468-474.

19. Kameyama K, Takami H (2003) Double parathyroid Carcinoma. Endocr J 50: 477-479.

20. Sato M, Miyauchi A, Namihira H, Bhuiyan MM, Imachi $\mathrm{H}$, et al. (2000) A newly recognized germline mutation of MEN1 gene identified in a patient with parathyroid adenoma and carcinoma. Endocrine 12: 223-226.

21. Ito T, Igarashi H, Uehara H, Berna MJ, Jensen RT (2013) Causes of death and prognostic factors in multiple endocrine neoplasia type 1: a prospective study: comparison of 106 men1/zollinger-ellison syndrome patients with 1613 literature men1 patients with or without pancreatic endocrine tumors. Medicine (Baltimore) 92: 135-181.

22. Almeida MQ, Stratakis CA (2010) Solid tumors associated with multiple endocrine neoplasias. Cancer Genet Cytogenet 203: 30-36.

23. Thakker RV, Newey PJ, Walls GV, Bilezikian J, Dralle H, et al. (2012) Clinical Practice guidelines for multiple endocrine neoplasia type 1 (MEN1). J Clin Endocrinol Metab 97: 2990-3011. 